\title{
IDENTIFICATION OF EXUDATES USING FUZZY MATHEMATICAL MORPHOLOGY
}

\author{
${ }^{1}$ Kittipol Wisaeng, ${ }^{1}$ Nualsawat Hiransakolwong and ${ }^{2}$ Ekkarat Pothiruk \\ ${ }^{1}$ Department of Computer Science, King Mongkut's Institute of Technology Ladkrabang, Bangkok 10520, Thailand \\ ${ }^{2}$ Ophthalmology Unit, Khonkaen Hospital, Khonkaen 40000, Thailand
}

Received 2013-12-06; Revised 2013-12-24; Accepted 2014-01-18

\begin{abstract}
Diabetic Retinopathy is the damage to the retina caused by complication and the most common cause of blindness in Thailand. Retinal image is essential for expert ophthalmologists to diagnose diseases. Several of method can achieve good performance on retinal feature are clearly visible. Unfortunately, the color retinal image in Thailand are low-resolution images. The existing method cannot identified lowresolution image. Therefore, this study is part of a larger effort to develop a new method for identification of exudates in low-resolution retinal image. In this study a fuzzy mathematical morphology based on fuzzy logical operator and mathematical morphology method is presented. The color retinal image are segmented by using fuzzy logical operator following key preprocessing step, i.e., color normalization, contrast enhancement, noise removal and color space selection. Afterward, a segmentation using mathematical morphology method was applied in this step. This enables its difference in our methods compared to other approach and the methods can achieve good performance even on lowresolution retinal images. Respect to the experimental results, the results obtained with fuzzy mathematical morphology better than the ones obtained with the fuzzy logical operator only method.
\end{abstract}

Keywords:Diabetic Retinopathy, Fuzzy Mathematical Morphology, Retinal Image, Expert Ophthalmologists, Exudates

\section{INTRODUCTION}

Diabetic retinopathy is a microvascular complication of diabetes, causing abnormalities in the retina. Exudates represent leak from surrounding capillaries and microaneurysms in diabetic retinopathy. The rapid increase of exudates pushes the limits of the exudates screening and semi-automatic retinal image analysis methods provide a potential solution. Unfortunately, the color retinal image in Thailand are low-resolution images. Thus, color retinal image segmentation in low-resolution images is difficult; take a long time and the expensive computational cost has become a bottleneck that limits clinical application. Serveral techniques have been employed to identification of exudates.
The thresholding and region growing technique are widely used. Kavitha and Devi (2005) proposed median filtering and morphology operation for vascular identification. Mul-tilevel threshoding is used to segment bright regions assumed to be the exudates or optic disc. They detec the optic disc as the converging point of vascular and then classifiy the other bright regions as exudates. Li and Chutatape (2003) used region growing and edge detection of exudates. Usher et al. (2004) identified the exudates region by using a region growing followed with edge detection techniques. The application failed to identified lowresulotion image and small exudates. Fuzzy logical operator has also been proposed as a possible solution to identification of exudates problem. Zhang and Corresponding Author: Kittipol Wisaeng, Department of Computer Science, King Mongkut's Institute of Technology Ladkrabang, Bangkok 10520, Thailand 
Chutatape (2005) segmented exudates by using fuzzy in Luv value. Osareh et al. (2001) used fuzzy c-mean clustering to detect digital retinal image and then used neural network train dataset and sparate exudates and non-exudates area by support vector machine. Gardner et al. (1996) used back propagation neural network to automated identification of diabetic retinopathy. Comparative the results by neural network method with of the expert ophthalmologist, the neural network achieved a sensitivity and specificity for the identification of diabetic retinopathy are 88.4 and $83.5 \%$, respectively. The techniques did not work well on low-resolution quality image. All methodologies described have some limitation. The approaches based in identification of exudates by machine learning need a long trianing time and it becomes computationally too heavy. Morever, the simple method for identification of exudates does not enable to detect the exudates boundary.

From the above studies, identification of exudates can be broken down to two situations: Low-resolution image and multimodal image (Fig. 1). The existing algorithm can achieve good performance when these two situations are not combined together. For example, it is difficult to identification of exudates an images taken at different times and different fields of view. To solve this problem, we proposed a new method for identification of exudates using fuzzy mathematical morphology method to segment and identification of exudaes in low-resolusion and multimodal image.

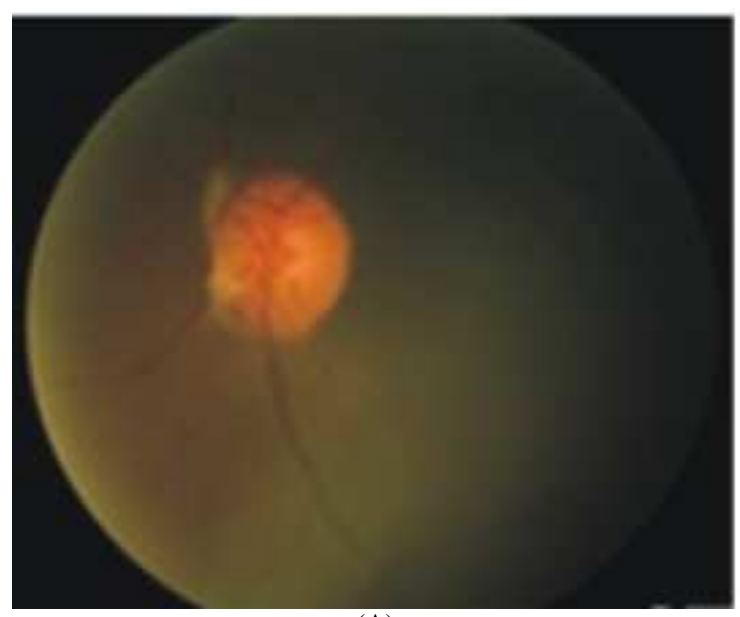

(A)

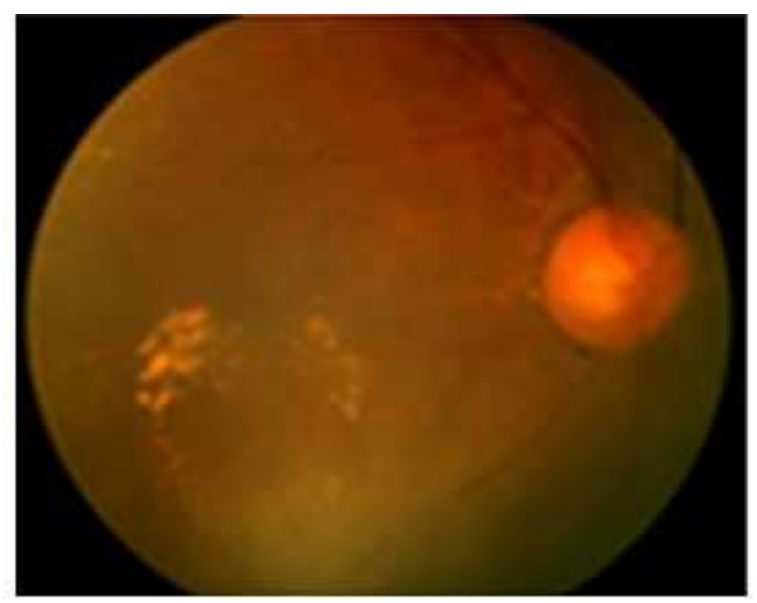

(B)

Fig. 1. Example of low resolution image and multimodal image taken from the same patients (A) typical normal image (B) abnormal image indicates exudates 


\section{MATERIALS AND METHODS}

\subsection{Patients}

The database consists of 2,084 retinal images, of which 968 at signs of exudates and 1,116 images do not contain any signs of exudates according to ophthalmologists participated in the annotation. The retinal images were taken by the local hospital with single degree 40 degree field of view. The image resolution is $700 \times 500$ pixels at 24 bits per pixel. All color retinal images are tested on a Core2 Duo $1.60 \mathrm{GHz}$ using MATLAB for algorithms. The overall procedure identification of exudates demonstrated in Fig. 2.

\subsection{Data Preparation}

In source image, the retinal image is created by emitting a flash lamp produced light into patient's eye through complex retina camera optics and capturing the reflected light to the camera sensor. One of the main problem for identification of exudates is the wide variability in the color of retinal image from different patients and different time. Thus, the color of exudates in some region of an images may appear dimmer than the background color of other regions. To solving these problem, we put retinal image through four preprocessing steps before commencing the identification of exudates. In the first step is to normalize the color of the source images across the database. We used histogram matching applied to each individual RGB values to histogram matches that of another image in database. A common application of this is to match the images from two sensors with slightly different responses, or from a sensor whose response changes over time. The following is an example using a greyscale image. The first image is the reference image and the histogram is given. The second image is the one whose histogram will be matched to the first. The third shows the resulting matched image (Fig. 3A-F).
Considered from Fig. 3E, although this histogram matching is suitable for retinal image. However, the contrast decreases with the more distance of pixels from the center, especially in the periphery. The number of pixels in these areas may have negligible influence on the the computation of a global transformation whose shape does not necessarily guarantee the desired local enchancement (Giordano et al., 2006). The solution is to devise the center of the neighborhood region is then moved to an adjacent pixel location and the procedure is repeated. The second pre-processing step, we apply local contrast enchancement (Chang and $\mathrm{Wu}, 1998$ ) to a transformation of the values inside small windows in the image in a way that all values are distributed around the mean and show all possible intensities. The techniques of local contrast enchancement are described below.

Given each $\mathrm{f}$ in the initial image and a small $\mathrm{M} \times \mathrm{M}$ running window $\mathrm{w}$, then the image is filtered to produce the new image pixel $f(i, j)$ Equation (1):

$$
f(i, j)=255\left(\frac{\left[\Psi_{w}(f)-\Psi_{w}\left(f_{\text {min }}\right)\right]}{\left[\Psi_{w}\left(f_{\text {max }}\right)-\Psi_{w}\left(f_{\text {min }}\right)\right]}\right)
$$

where the $\psi$ is defined as Equation (2):

$$
\Psi_{\mathrm{w}}(\mathrm{f})\left[1+\exp \left(\frac{\langle\mathrm{f}\rangle_{\mathrm{w}}-\mathrm{f}(\mathrm{i}, \mathrm{j})}{\sigma_{\mathrm{w}}}\right)\right]^{-1}
$$

The $f_{\max }$ and $f_{\min }$ are referred as the whole image maximum and minimum intensity values; while $\langle\mathrm{f}\rangle_{\mathrm{w}}$ and $\sigma_{\mathrm{w}}$ indicate the local window mean and standard deviation within each window provides considerable contrast enhancement, which are defined as Equation (3):

$$
<\mathrm{f}>_{\mathrm{w}}=\frac{1}{\mathrm{M}^{2}} \sum_{(\mathrm{i}, \mathrm{j}) \in \mathrm{w}(\mathrm{k}, \mathrm{l})} \mathrm{f}(\mathrm{i}, \mathrm{j})
$$

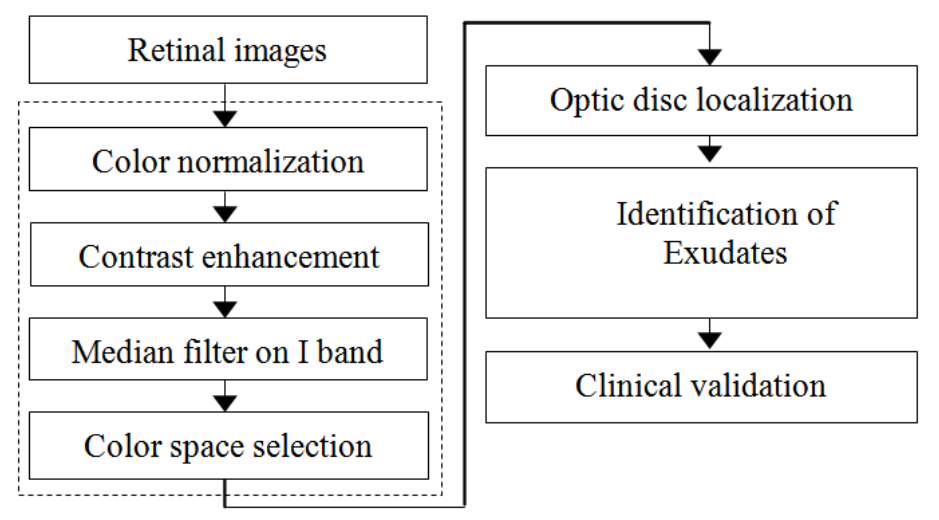

Fig. 2. The outline of our the proposed system for automatic identification of exdates 


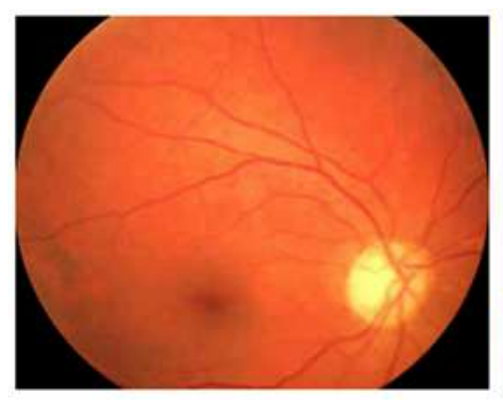

A

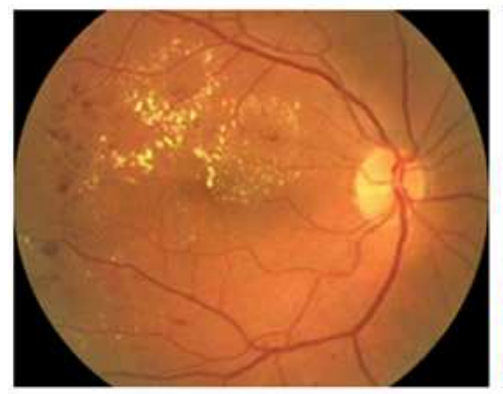

C

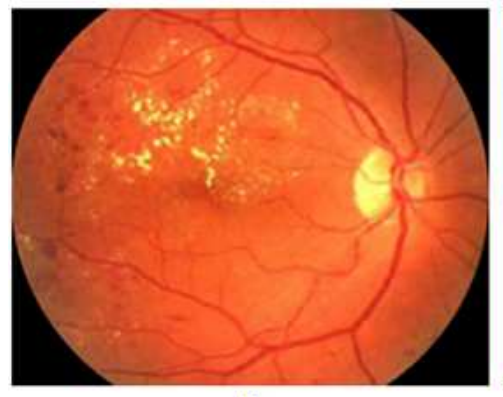

E

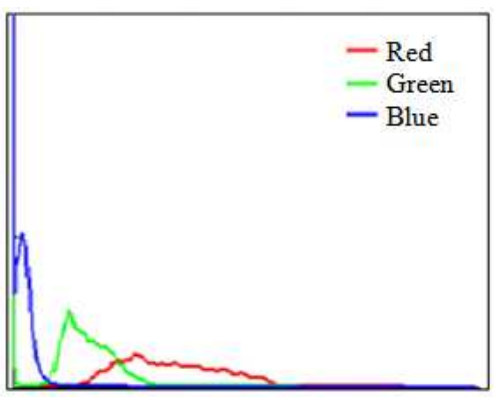

B

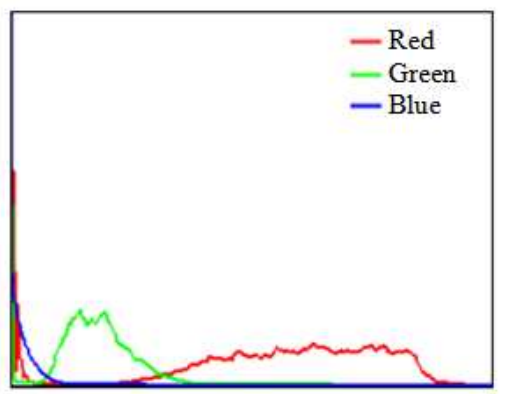

D

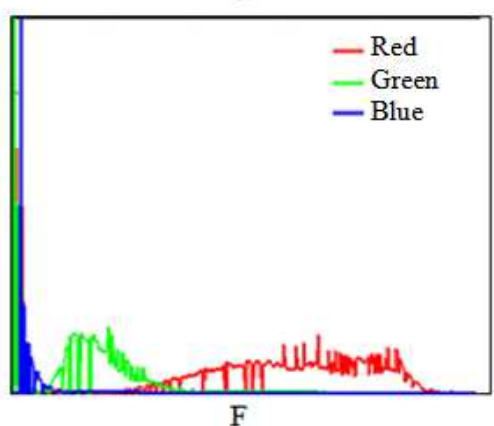

Fig. 3. The color normalization process based on histogram maching, (A) The reference image, (B) Histogram of reference image, (C) Image to be adjusted, (D) Histogram of image to be adjusted, (E) Histogram matched image (F) Histogram of matched image

$$
\sigma_{w}=\sqrt{\frac{1}{M^{2}} \sum_{(i, j) w(k, l)}\left(f(i, j)-\langle f\rangle_{w}\right)^{2}}
$$

where, $(\mathrm{k}, \mathrm{l})$ represents the location of each pixel within window $\mathrm{w}$. The size of window $\mathrm{M}$ should be chosen to be large enough to contain a statistically representative distribution of the local variations of pixels. On the other hand, it must be small enough to not be influenced by the gradual variation of the contrast between the retinal image center and the periphery (Osareh et al., 2003). Here, the window size was empirically set to $69 \times 69$ for our processing, although the other values may be also appropriate. The exponential function (Equation 4) produces significant enhancement when the contrast is low, while it provides less enhancement if the contrast is already high. The examples of retinal images after the contrast enhancement (Osareh et al., 2009). While the contrast enhancement improves the contrast of exudates, it may also enhance the contrast of some non-exudates background pixels, so that these pixels can wrongly be identified as exudates. The third preprocessing step, we used median filtering to remove or reduce noise in an image. The benefit of median filtering operation is simultaneously reducing noise and preserving edges. The median filter considers each pixel in the image in turn and looks at its nearby neighbors to decide 
whether or not it is representative of its surroundings. Instead of simply replacing the pixel value with the mean of neighboring pixel values, it replaces it with the median of those values. The median is calculated by first sorting all the pixel values from the surrounding neighborhood into numerical order and then replacing the pixel being considered with the middle pixel value. Figure $\mathbf{4}$ and 5 illustrates an example of median filtering and example calculation. Finally, Red, RGB vlaue of original retinal image is transformed to Luv color space.

\subsection{Detection of Optic Disc}

Identification of optic disc plays an important role in detect of exudates. However, the optic disc is similar intensity with exudates. Therefore, to prevent the optic disc from interfering with exudates, our proposed a new method to detection of optic disc before identification of exudates (Wisaeng et al., 2013).

\subsection{Identification Using Fuzzy Logical Operator}

The fuzzy logical operator is a data clustering algorithm in which each data point belongs to a cluster, to a degree specified by a membership grade. Cannon et al. (1986) originally introduced this technique. Fuzzy partitions a collection of $\mathrm{n}$ vectors, $c_{j}, j=1, \ldots, n$, into c fuzzy groups and finds a cluster center in each group, such that a cost function based on a distance function is minimized. Because of the fuzzy partition, a given data point can belong to several groups with a degree of membership specified by a grade between 0 and 1 , such that the constrains in Equation (5) are statisfied, (Gil et al., 1999). These values are collected in a membership matrix, $U$. However, imposing normalization stipulates that the summation of degrees of memberships for a data set must always be equal to unity (Homaeinezhad et al., 2011):

$$
\sum_{\mathrm{i}=1}^{\mathrm{c}} \mathrm{u}_{\mathrm{ij}}=1, \forall \mathrm{j}=1, \ldots, \mathrm{n}
$$

The cost function or objective function for fuzzy logical operator is:

$$
\mathrm{J}_{\mathrm{m}}\left(\mathrm{U}, \mathrm{c}_{1}, \ldots, \mathrm{c}_{\mathrm{c}}\right)=\sum_{\mathrm{j}=1}^{\mathrm{N}} \sum_{\mathrm{i}=1}^{\mathrm{C}}\left(\mathrm{u}_{\mathrm{ij}}\right)^{\mathrm{m}}\left\|\mathrm{x}_{\mathrm{i}}-\mathrm{c}_{\mathrm{j}}\right\|_{\mathrm{A}}^{2}
$$

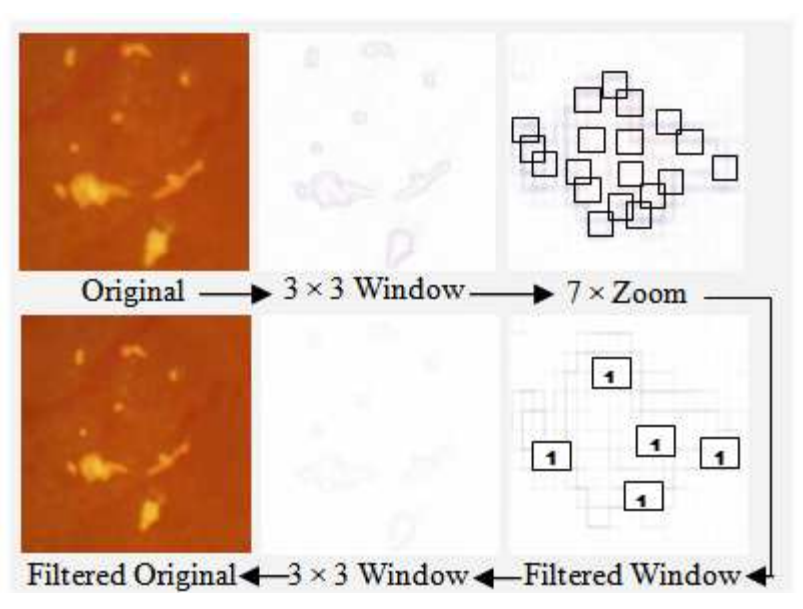

Fig. 4. Median filtering example

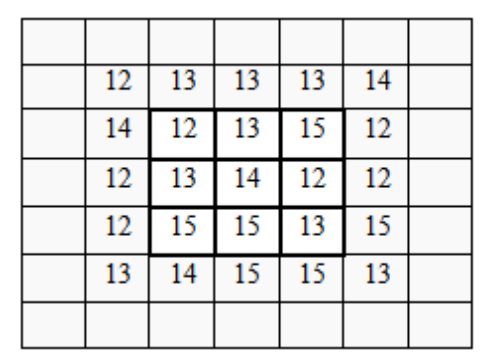

Neighbourhood Values: $15,13,12,15,14$, $13,13,12,15$

Median Value: 13

Fig. 5. Calculating the median value of a pixel neighborhood. As can be seen, the central pixel value of 15 is rather unrepresentative of the surrounding pixels and is replaced with the median value: 13 . A $3 \times 3$ square neighborhood is used

Where:

$\mathrm{m}=$ An exponential weighting function that controls the fuzziness of the membership function

$\mathrm{N}=$ Number of features, 5 for our case

$\mathrm{C}=$ Number of cluster, $\mathrm{u}_{\mathrm{ij}}$ is the degree of membership of $\mathrm{x}_{\mathrm{i}}$ in the cluster $\mathrm{j}$

$\mathrm{x}_{\mathrm{i}}=$ The ith of d-dimensional measured data

$c_{j}=$ The d-dimension center of the cluster and

$|\cdot|=$ The Euclidean distance between the ith cluster and the jth data point

The squared distance between ith and jth shown in Equation (6) is computed in A-norm via Equation (7).

$d_{i j}^{2}=\left\|x_{i}-c_{j}\right\|_{A}^{2}=\left(x_{i}-c_{j}\right)^{T} A\left(x_{i}-c_{j}\right)$ 
With these observations, we can decompose $\mathrm{J}_{\mathrm{m}}$ into its basic elements to see what property of the points $\left(\mathrm{x}_{\mathrm{i}}\right)$ is measures Equation (8a to $\left.\mathrm{d}\right)$ :

$\mathrm{d}_{\mathrm{ij}}^{2}=$ squared A-distance

frompoint $\mathrm{x}_{\mathrm{i}}$ to center of $\mathrm{c}_{\mathrm{j}}$

$\left(\mathrm{u}_{\mathrm{ij}}\right)^{\mathrm{m}} \mathrm{d}_{\mathrm{ij}}^{2}=$ squared A-error incurred by representing

$\mathrm{x}_{\mathrm{i}}$ by $\mathrm{c}_{\mathrm{j}}$ weighted by the membership of $\mathrm{x}_{\mathrm{i}} \mathrm{in}$ cluster $\mathrm{j}$

$\sum_{\mathrm{i}=1}^{\mathrm{c}}\left(\mathrm{u}_{\mathrm{ij}}\right)^{\mathrm{m}} \mathrm{d}_{\mathrm{ij}}^{2}=$ sum of squared A-error due

to $\mathrm{x}_{\mathrm{i}}$ partial replacement by all $\mathrm{C}$ of the center $\left(\mathrm{c}_{\mathrm{j}}\right)$

$\sum_{j=1}^{N} \sum_{i=1}^{C}\left(u_{i j}\right)^{m} d_{i j}^{2}=$ overall weight

sumof generalizedA - error

Fuzzy partitioning is carried out through an iterative optimization of the objective function shown above, with the update of membership $u_{i j}$ and the cluster centers $c_{j}$ by using Equation (9 and 10), respectively:

$$
\begin{gathered}
\mathrm{u}_{\mathrm{ij}}=\frac{1}{\sum_{\mathrm{k}=1}^{\mathrm{c}}\left(\frac{\mathrm{d}_{\mathrm{ij}}}{\mathrm{d}_{\mathrm{kj}}}\right)^{2 /(\mathrm{m}-1)}} \\
\mathrm{c}_{\mathrm{j}}=\frac{\sum_{\mathrm{i}=1}^{\mathrm{N}} \mathrm{u}_{\mathrm{ij}}^{\mathrm{m}} \mathrm{x}_{\mathrm{i}}}{\sum_{\mathrm{j}=1}^{\mathrm{N}} \mathrm{u}_{\mathrm{ij}}^{\mathrm{m}}}
\end{gathered}
$$

This iteration will stop when Equation (11) is satisfied:

$$
\max _{\mathrm{ij}}\left(\left|\mathrm{u}_{\mathrm{ij}}^{(\mathrm{k}+1)}-\mathrm{u}_{\mathrm{ij}}^{(\mathrm{k})}\right|\right)<\mathrm{T}
$$

Where:

$\mathrm{T}=$ A termination criterion

$\mathrm{K}=$ The iteration number

This procedure converges to a local minimum or a saddle point of $\mathrm{J}$. The input to the fuzzy logical operator is a set of feature. The number of cluster is required at the beginning and the algorithm is proposed by (Sopharak et al., 2009) and composed following step:
Step 1: Initialize the fuzzy partition matrix $U=$ $\left(\mathrm{u}_{\mathrm{ij}}\right)\left(\mathrm{U}^{(0)}\right)$ by generating random numbers in the range 0-1 subject to Equation (12):

$\sum_{\mathrm{i}=1}^{\mathrm{N}} \sum_{\mathrm{j}=1}^{\mathrm{C}} \mathrm{u}_{\mathrm{ij}}=1$

Step 2: At k-step; calculate the centers vectors $C^{(\mathrm{k})}=\left(\mathrm{c}_{\mathrm{j}}\right)$ with $\left(\mathrm{U}^{(\mathrm{k})}\right)$ according to Equation (10).

Step 3: Update the fuzzy partition matrix $\mathrm{U}^{(\mathrm{k})}, \mathrm{U}^{(\mathrm{k}+1)}$ by the new computed $\mathrm{u}_{\mathrm{ij}}$ according to Equation (19).

Step 4: Compute the objective function according to Equation (6). If the difference between adjacent values of the objective function is less than Termination criterion (T) the stop the iteration; otherwise return to step 2 .

\subsection{Identification Using Mathematical Morphology Method}

The resulting image from segmentation by fuzzy logical operator is assign as input to the segmentation by using mathematical morphology method. The mathematical morphology method by dilation was applied on the previous overlay image (The resulting segmentation from fuzzy logical operator). A simple structuring that can be used for repairing the gaps until the resulting of dilation the original image with structuring element have been bridging. The dilation can be computed by using Equation (13). With $\mathrm{A}$ and $\mathrm{B}$ as sets in $\mathrm{x}^{2}$, the dilation of $\mathrm{A}$ by $\mathrm{B}$, denoted $\mathrm{A} \oplus \mathrm{B}$, is defined as:

$\mathrm{A} \oplus \mathrm{B}=\left\{\mathrm{x} \mid(\mathrm{B})_{\mathrm{x}} \cap \mathrm{A} \neq \varnothing\right\}$

where, $\mathrm{A} \oplus \mathrm{B}$ is the resultant picture of the dilation of $\mathrm{A}$ with $\mathrm{B}$.

This equation is baed on obtaining the reflection of $\mathrm{B}$ about its origin and shifting this reflection by $\mathrm{x}$. The dilation of A by $B$ then is the set of all displacements, $\mathrm{x}$, such that $\mathrm{B}$ and A overlap by at least one element. Based on this interpretation, Equation (13) may be rewritten as Equation (14):

$\mathrm{A} \oplus \mathrm{B}=\left\{\mathrm{x} \mid\left[(\mathrm{B})_{\mathrm{x}} \cap \mathrm{A}\right] \subseteq \mathrm{A}\right\}$

Set B is commonly referred to as the structuring element in dilation, as well as in other morphological operations. In matlab, dilation can be implemented by a function, called imdilate. (Satisfying, MorphPic = imdilate (OriginPic, SE); MorphPic, is the 
morphological picture, OriginPic, the resultant picture and $\mathrm{SE}$, is the structuring element.

\subsection{Performance Measurement}

To automatic pixel based evaluation methods performance, the pixel based evaluation protocal is to generate a sensitivity (SE), specificity (SP) and accuracy (AC) score values of the test image (Satisfying, $\mathrm{SE}=\mathrm{TP} / \mathrm{TP}+\mathrm{FN}, \mathrm{SE}$ corresponds to the proportion of correctly diagnosed exudates test subjects among all exudates test subjects, $\mathrm{SP}=\mathrm{TN} / \mathrm{TN}+\mathrm{FP}, \mathrm{SP}$ corresponds to the proportion of correctly diagnosed non-exudates test subjects among all non-exudates test subjects and $\mathrm{AC}=\mathrm{TP}+\mathrm{TN} / \mathrm{TP}+\mathrm{FP}+\mathrm{FN}+\mathrm{TN}, \mathrm{AC}$ is overall per-pixel success rate of the methods).

For exudates test subject, the result is True Positive (TP) if the diagnostic test outcome is exudates and False Negative (FN) if the diagnostic test outcome is non-exudates. For non-exudates test subject, the result is true negative if the diagnostic test outcome is nonexudates and false positive if the diagnostic test outcome is exudates (Tomi et al., 2011).

\section{RESULTS AND DISCUSSION}

Many experiments have been performed on exudates and non-exudates retinal images to test and validate our method. The result from the fuzzy logical operator was validated using 2,084 images with their clinician hand-labelled images in the pixel level with $96.7,71.4$ and $79 \%$ sensitivity, specificity and accuracy respectively. Figure 6 shows a simulated view which an exudates using $n=8$. Afterward, The resulting segmentation from the fuzzy logical operator is assign as input to the segmentation by mathematical morphology method. The average values of sensitivity, specificity and accuracy of validation results. The fuzzy mathematical morphology successfully with sensitivity, specificity and accuracy of $92.06,92.92$ and $92.49 \%$, respectively. From simulated experiments results, if used fuzzy logical operator only; it gives a high TP value with a high FP value. Using fuzzy mathematical morphology, gives higher accuracy with a lower FP value.

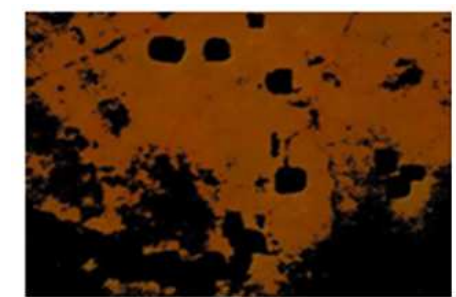

(A)

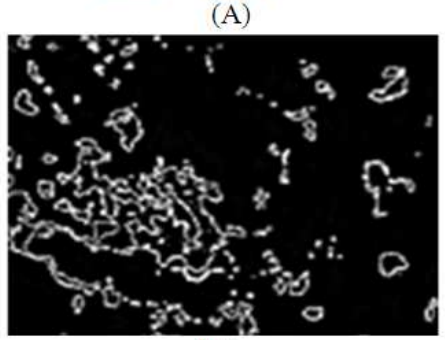

(D)

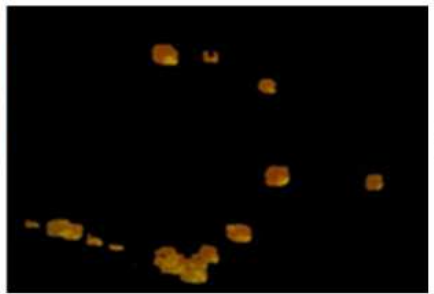

(B)

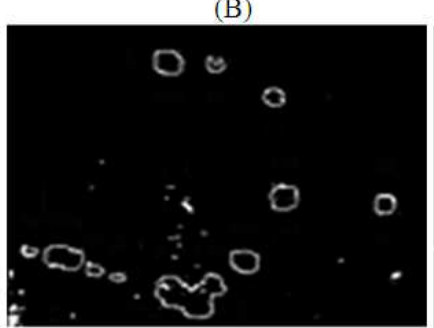

(E)

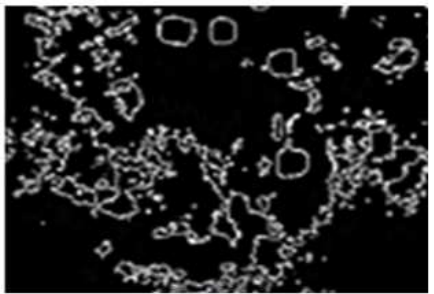

(C)

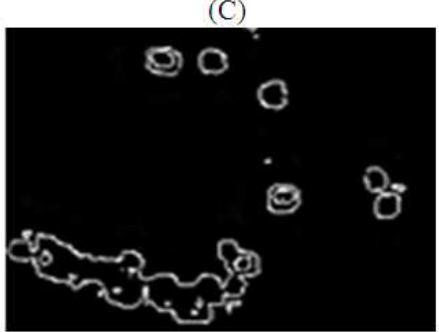

(F)

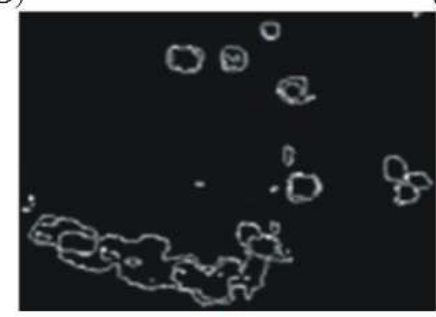

(G)

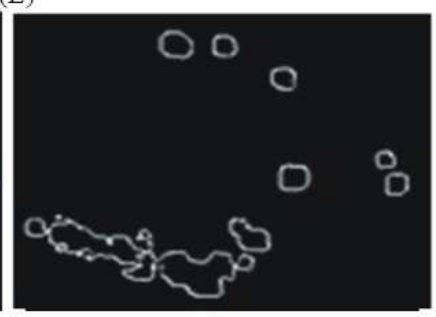

(H)

Fig. 6. The fuzzy logical operator results with $n=8$, (A) $n=1,(B) n=2,(C) n=3$, (D) $n=4$, (E) $n=5,(F) n=6$, (G) $n=7$, (H) $n=8$ 


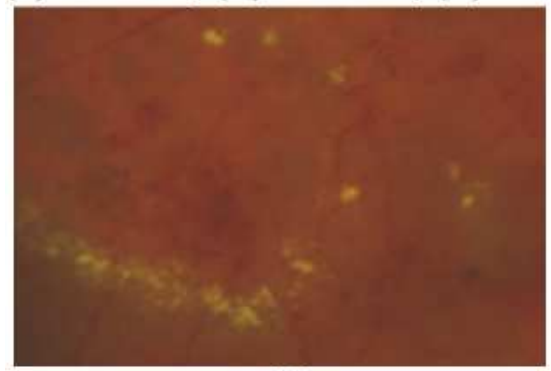

(A)

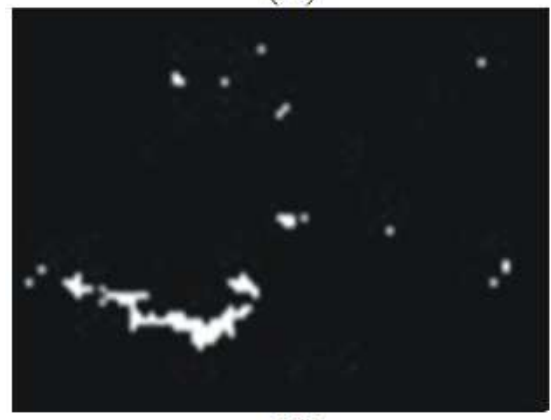

(C)

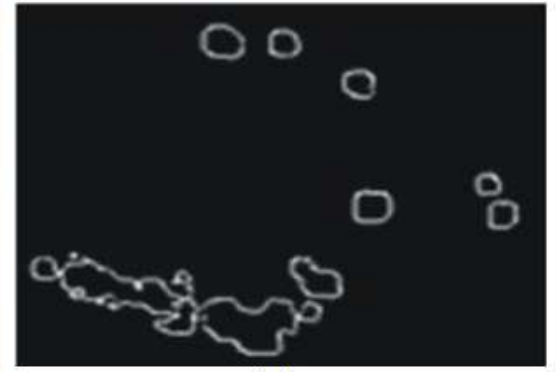

(B)

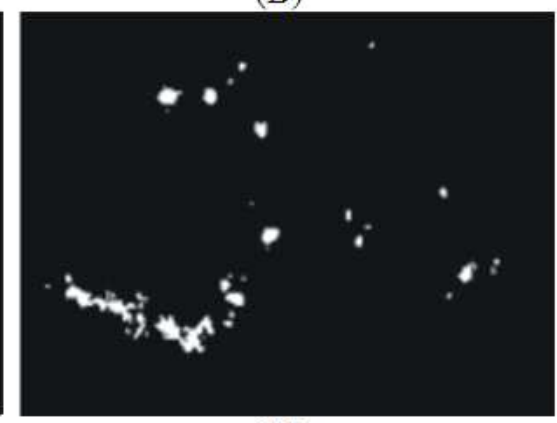

(D)

Fig. 7. The results for identification of exudates, (A) original image, (B) segmentation using fuzzy logical operator, (C) segmentation using fuzzy mathematical morphology and (E) manually labeled by expert ophthamologist

The baseline algorithm, the results from the fuzzy mathematical morphology achieved accuracy better than fuzzy logical operator only technique very clear. The results from the fuzzy mathematical morphology and ground-truth image are displays in Fig. 7.

\section{CONCLUSION}

There were two main issues that were addressed in this papers: (1) how good the identification of exudates in low-resolution. (2) how the performance of the identification of exudates should be evaluated to produce reliable. In addition, a robust and automated detection of optic disc method using Ostu's method and morphological method was presented as a supplementary item.

In this study, we have proposed a new methods to automatically identified of exudates from low-resolution images and multimodal images taken from diabetic retinopathy. The study is based on the fuzzy logical operator segmentation and mathematical morphology. Five characteristics of exudates from preprocessing step, namely, retinal color normalization, local contrast enhancement, median filtering, RGB color space was transformed to Luv values and detection of optic disc are selected to input feature. The performance of methods is measured against expert ophthalmologist hand-drawn ground-truth. The sensitivity, specificity and accuracy are also used to evaluate the method. The result shows that accuracy values increase when combine the fuzzy logical operator and mathematical morphology. Overall, the simulated experiments results show that careful preprocessing step and appropriate method together provide identification of exudates performance even on low-resolution images and multimodal images.

\section{REFERENCES}

Cannon, R.L., J.V. Dave and J.C. Bezdek, 1986. Efficient implementation of the fuzzy c-means clustering algorithms. IEEE Trans. Patt. Anal. Mach. Intell., $\quad 8$ : 248-255. DOI: 10.1109/TPAMI.1986.4767778

Chang, C.D. and W.R. Wu, 1998. Image contrast enhancement based on a histogram transformation of local standard deviation. IEEE Trans. Med. Imag., 17: 518-531. DOI: 10.1109/42.730397

Gardner, G.G., D. Keating, T.H. Williamson and A.T. Elliott, 1996. Automatic detection of diabetic retinopathy using an artificial neural network: A screening tool. Br. J. Ophthalmol., 80: 940-944. DOI: $10.1136 /$ bjo.80.11.940 
Gil, M., E.G. Sarabia, J.R. Llata and J.P. Oria, 1999. Fuzzy c-means clustering for noise reduction, enhancement and reconstruction of 3D ultrasonic images. Proceedings of the 7th IEEE International Conference on Emerging Technologies and Factory Automation, Oct. 18-21, IEEE Xplore Press, Barcelona, pp: 465-472. DOI: 10.1109/ETFA.1999.815392

Giordano, D., R. Leonardi, F. Maiorana and C. Spampinato, 2006. Cellular neural networks and dynamic enhancement for cephalometric landmarks detection. Artif. Intell. Soft Comput., 4029: 768777. DOI: 10.1007/11785231_80

Homaeinezhad, M.R., E. Tavakkoli and A. Ghaffari, 2011. Discrete wavelet-based fuzzy network architecture for ECG rhythm-type recognition: Feature extraction and clustering-oriented tuning of fuzzy inference system. Int. J. Signal Process. Image Process. Patt. Recog., 4: 107-130.

Kavitha, D. and S.S. Devi, 2005. Automatic detection of optic disc and exudates in retinal images. Proceedings of the International Conference on Intelligent Sensing and Information, Jan. 4-7, IEEE Xplore Press, pp: 502-506. DOI: 10.1109/ICISIP.2005.1529506

Li, H. and O. Chutatape, 2003. A model-based approach for automated feature extraction in fundus images. Proceedings of the 9th IEEE International Conference on Computer Vision, Oct. 13-16, IEEE Xplore Press, Nice, France, pp: 394-399. DOI: 10.1109/ICCV.2003.1238371

Osareh, A., B. Shadgar and R. Markham, 2009. A computational-intelligence-based approach for detection of exudates in diabetic retinopathy images. IEEE Trans. Inform. Technol. Biomed., 13: 535545. DOI: 10.1109/TITB.2008.2007493
Osareh, A., M. Mirmehdi, B. Thomas and R. Markham, 2001. Automatic recognition of exudative maculopathy using fuzzy c-means clustering and neural networks. Proceedings of the Medical Image Understanding Analysis Conference, (MIUAC' 01), pp: 49-52.

Osareh, A., M. Mirmehdi, B. Thomas and R. Markham, 2003. Automated identification of diabetic retinal exudates in digital colour images. Br. J. Ophthalmol., 87: 1220-1223.

Sopharak, A., B. Uyyanonvara and S. Barman, 2009. Automatic exudate detection from non-dilated diabetic retinopathy retinal images using fuzzy cmeans clustering. Sensors, 9: 2148-2161. DOI: $10.3390 / \mathrm{s} 90302148$

Tomi, K., J.K. Kamarainen, L. Lensu, H. Kalviainen and H. Uusitalo, 2011. Detection and decision-support diagnosis of diabetic retinopathy using machine vision. Patt. Recog. Image Anal., 21: 140-143. DOI: 10.1134/S1054661811020465

Usher, D., M. Dumskyj, M. Himaga, T.H. Williamson and S. Nussey et al., 2004. Automated detection of diabetic retinopathy in digital retinal images: A tool for diabetic retinopathy screening. Diabet. Med., 21: 84-90. PMID: 14706060

Wisaeng, K., N. Hiransakolwong and E. Pothiruk, 2013. Automatic detection of retinal exudates using a support vector machine. Applied Med. Inform., 32: 33-41.

Zhang, X. and O. Chutatape, 2005. Top-down and bottom-up strategies in lesion detection of background diabetic retinopathy. Proceedings of the IEEE Computer Society Conference on Computer Vision and Pattern Recognition, Jun. 20-25, IEEE Xplore Press, pp: 422-428. DOI: 10.1109/CVPR.2005.346 